\title{
Anti-Inflammatory Potential of Probiotic Strain Weissella cibaria JW15 Isolated from Kimchi through Regulation of NF-KB and MAPKs Pathways in LPS-Induced RAW 264.7 Cells
}

\author{
Hyung-Seok Yu ${ }^{1}$, Na-Kyoung Lee ${ }^{1}$, Ae-Jin Choi ${ }^{2}$, Jeong-Sook Choe ${ }^{2}$, Chun Ho Bae ${ }^{3}$, and Hyun-Dong Paik ${ }^{\text {* }}$ \\ ${ }^{1}$ Department of Food Science and Biotechnology of Animal Resources, Konkuk University, Seoul 05029, Republic of Korea \\ ${ }^{2}$ Functional Food and Nutrition Division, Department of Agrofood Resources, National Academy of Agricultural Science, Rural Development \\ Administration, Jeollabuk-do 55365, Republic of Korea \\ ${ }^{3}$ Aram Co., Ltd., Gyeonggi-do 12735, Republic of Korea
}

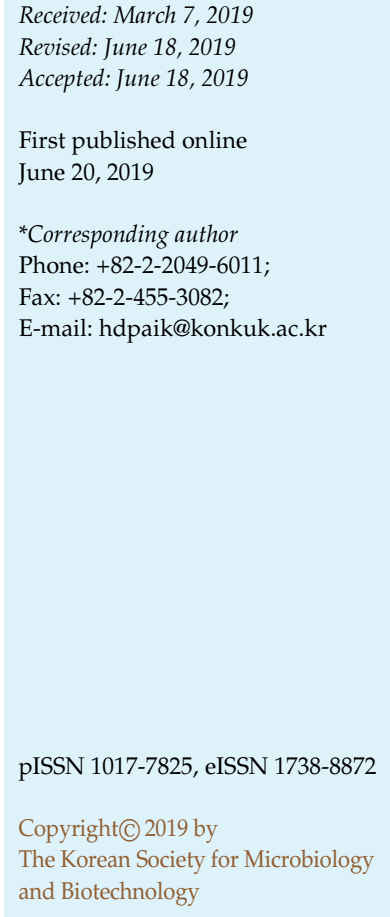

Probiotics are known to provide the host with immune-modulatory effects and are therefore of remarkable interest for therapeutic and prophylactic applications against various disorders, including inflammatory diseases. Weissella cibaria JW15 (JW15) has been reported to possess probiotic and antioxidant properties. However, the effect of JW15 on inflammatory responses has not yet been reported. Therefore, the objective of the current study was to evaluate the anti-inflammatory potential of JW15 against lipopolysaccharide (LPS) stimulation. The production of pro-inflammatory factors and the cellular signaling pathways following treatment with heat-killed JW15 was examined in LPS-induced RAW 264.7 cells. Treatment with heat-killed JW15 decreased nitric oxide and prostaglandin $\mathrm{E}_{2}$ production via downregulation of the inducible nitric oxide synthase and cyclooxygenase-2. In addition, treatment with heat-killed JW15 suppressed the expression of pro-inflammatory cytokines, interleukin (IL)-1 $\beta$, IL-6, and tumor necrosis factor- $\alpha$. The anti-inflammatory properties of treating with heat-killed JW15 were associated with mitogen-activated protein kinase signaling pathwaymediated suppression of nuclear factor- $\mathrm{kB}$. These results indicated that JW15 possesses antiinflammatory potential and provide a molecular basis regarding the development of functional probiotic products.

Keywords: Weissella cibaria JW15, kimchi, probiotics, anti-inflammation, nuclear factor-кB, mitogen-activated protein kinase

\section{Introduction}

Inflammation is a basic host defense response against external invasions and is necessary for the restoration and maintenance of health [1]. Macrophages, a type of innate immune cell, predominantly mediate inflammatory responses through phagocytosis and release of proinflammatory molecules that include cytokines, such as interleukin (IL)-1 $\beta$, IL-6, and tumor necrosis factor (TNF)- $\alpha$. [2]. The activation of macrophages is triggered by various stimuli including lipopolysaccharides (LPS), which are endotoxins originated from Gram-negative pathogens, and results in the activation of inflammation-related cellular signaling pathways, such as the mitogen-activated protein kinases (MAPKs) and nuclear factor (NF)- $\mathrm{B}$ [3]. The activation of MAPKs and NF- $\mathrm{BB}$ up-regulates the expression of inflammatory genes and is followed by production of pro-inflammatory mediators including cytokines, nitric oxide $(\mathrm{NO})$ and prostaglandin $\mathrm{E}_{2}\left(\mathrm{PGE}_{2}\right)$. However, prolonged or excessive expression of those inflammatory mediators may result in inflammatory disorders or cancer [4].

As the largest portion of the microbiome is the gastrointestinal tract (GIT), the intestinal microbiota of the host is the primary source of microbe-derived beneficial 
effects [5]. Probiotics are viable microorganisms that confer health benefits such as regulation of gut microbiota balance and immunomodulatory effects on the host through colonization in the intestinal microflora. [6]. Therefore, the immunomodulatory potential of probiotics, especially lactic acid bacteria (LAB), is of continued interest for developing therapeutic and prophylactic treatments against diverse disorders, ranging from pathogen-associated diarrhea to allergic responses [7-9]. Accordingly, a growing number of studies have reported the immunomodulatory properties of probiotics, describing the Lactobacillusmediated modulation of Th1/Th2 polarization, attenuation of inflammatory bowel disease (IBD), and suppression of pro-inflammatory cytokines against LPS stimulation [10, 11].

Kimchi, a traditional Korean fermented vegetable side dish, is commonly regarded as a health-enhancing functional food due to its diverse bio-active components such as vitamins, flavonoids, polyphenols, and LAB. Various LAB, including Leuconostoc spp., Lactobacillus spp., and Weissella spp., are associated with the fermentation of kimchi [12]. Weissella cibaria, which was recently classified as LAB, has been derived from fermented foods, and is known to be a typically dominant strain of kimchi microflora along with Leuconostoc mesenteroides and Lactobacillus plantarum [13]. In addition, $W$. cibaria has been reported to possess probiotic potential and various beneficial properties, including functional exopolysaccharide production and attenuation of pathogen-mediated inflammatory responses. Accordingly, previous studies have demonstrated that $W$. cibaria JW15 (JW15) isolated from kimchi exhibits probiotic, antioxidant, and immuno-stimulatory properties [14, 15].

Although such studies have reported on the functional properties of $W$. cibaria strains, the effects of $W$. cibaria strains against LPS-induced inflammation have not yet been reported. Therefore, the present study aimed to evaluate the anti-inflammatory potential of JW15. The effect of heat-killed JW15 on the expression of proinflammatory mediators and the cellular signaling pathways was investigated in LPS-induced murine macrophage, RAW 264.7 cells.

\section{Materials and Methods}

\section{Chemicals and Reagents}

Dulbecco's modified Eagle's medium (DMEM), water, antibiotics, fetal bovine serum (FBS), and phosphate buffered saline (PBS) were purchased from HyClone Laboratories, Inc. (USA). An enzyme-linked immunosorbent assay (ELISA) kit was purchased from R\&D Systems (USA). Specific primers used in reverse transcription-polymerase chain reaction (RT-PCR) were purchased from Bionics (Korea). RIPA lysis buffer, Halt protease and phosphatase inhibitor cocktail, and PCR reagents were purchased from Thermo Scientific Pierce (USA). Primary antibodies against $\beta$-actin, inducible nitric oxide synthase (iNOS), and cyclooxygenase (COX)-2 were purchased from Thermo Scientific Pierce (USA). Primary antibodies for phosphorylated-extracellular signalregulated kinase (p-ERK) 1/2, ERK 1/2, p-c-jun N-terminal

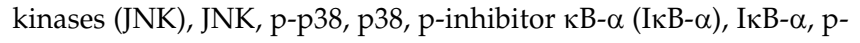
$\mathrm{p} 65$, and $\mathrm{p} 65$ and horseradish peroxidase (HRP)-conjugated secondary antibodies were purchased from Santa Cruz Biotechnology (USA). Materials and equipment for western blotting assays were purchased from Bio-Rad (USA), and other chemicals were purchased from Sigma-Aldrich (USA).

\section{Bacterial Strains and Sample Preparation}

Lactobacillus rhamnosus GG (KCTC 12202BP, LGG) and Weissella cibaria JW15 (KACC 91811P, JW15) was obtained from the Korean Collection for Type Cultures (Korea) and Korean Agricultural Culture Collection (Korea), respectively. LGG was employed for comparative analysis as a representative commercial probiotic strain. LGG and JW15 were cultivated and maintained in MRS broth (Difco Laboratories, USA) at $37^{\circ} \mathrm{C}$. To evaluate the antiinflammatory potential of these strains, overnight cultivated LGG and JW15 were heat-killed at $90^{\circ} \mathrm{C}$ for $30 \mathrm{~min}$. Following centrifugation $(12,000 \times g, 5 \mathrm{~min})$, cell pellets were rinsed thrice with PBS and suspended in DMEM to obtain concentrations of $6 \mathrm{Log} \mathrm{CFU} / \mathrm{ml}$ and $7 \mathrm{Log} \mathrm{CFU} / \mathrm{ml}$ by adjusting the absorbance at $600 \mathrm{~nm}$.

\section{Cell Culture}

The RAW 264.7 cell line, a murine macrophage, was obtained from the Korean Cell Line Bank (Korea) and maintained in DMEM supplemented with $10 \%$ FBS and $1 \%$ antibiotics $(100 \mathrm{U} / \mathrm{ml}$ penicillin and $100 \mu \mathrm{g} / \mathrm{ml}$ streptomycin) at $37^{\circ} \mathrm{C}$ in an atmosphere of $5 \% \mathrm{CO}_{2}$. Cells were sub-cultured and plated at $80-90 \%$ of confluency.

\section{Sample Treatment}

To evaluate the anti-inflammatory potential of LAB strains, RAW 264.7 cells were pre-incubated in 96-well plates $\left(2.0 \times 10^{5}\right.$ cells/well) and 6-well plates $\left(1.0 \times 10^{6}\right.$ cells/well $)$ for $2 \mathrm{~h}$ and $18 \mathrm{~h}$, respectively. And then, cells were treated with different concentrations $(0,6$, and $7 \mathrm{Log} \mathrm{CFU} / \mathrm{ml})$ of heat-killed LGG or JW15. After $2 \mathrm{~h}$ of incubation, cells were stimulated with or without LPS $(1 \mu \mathrm{g} / \mathrm{ml})$ for $24 \mathrm{~h}$. To investigate the effect of LAB strains on the MAPKs and NF- $\kappa B$ activation, cells were stimulated with LPS for $30 \mathrm{~min}$.

\section{Measurement of NO Production}

The effect of LAB strains on the production of NO was determined in LPS-induced RAW 264.7 cells by using Griess reaction as described by Kim et al. [16] with slight modification. 
Cells plated in 96-well plates were treated with samples and LPS as described in the sample treatment section. Supernatant from each well was mixed with an equal volume of Griess reagent and placed in the dark for $10 \mathrm{~min}$ at room temperature. Absorbance of each well was measured at $540 \mathrm{~nm}$ using a microplate spectrophotometer (BioTek, USA). Nitrite levels in growth medium were calculated using a standard curve constructed using $\mathrm{NaNO}_{2}$ in DMEM.

\section{Determination of Cell Viability}

The effect of LAB strains on the viability of RAW 264.7 cells was assessed by using the 5-3-(4,5-dimethylthiazol-2-yl)-2,5diphenyltetrazolium bromide (MTT) assay according to described by Kim et al. [16] with minor modification. After aspiration of supernatant for determination of the NO, cells were treated with MTT solution (2.5 mg/ml in PBS) and incubated for $1 \mathrm{~h}$. Discarding the supernatant, dimethyl sulfoxide was added to each well and generated formazan deposits were dissolved. Absorbance of each well was measured at $570 \mathrm{~nm}$ using a microplate spectrophotometer. The cell viability was calculated as a percentage of the absorbance compared to LPS-positive groups.

\section{Assessment of $\mathrm{PGE}_{2}$ and Pro-Inflammatory Cytokines}

The effect of $\mathrm{LAB}$ strains on the production of $\mathrm{PGE}_{2}$ and proinflammatory cytokines, IL- $1 \beta$, IL- 6 , and TNF- $\alpha$, was assessed in LPS-induced RAW 264.7 cells using an ELISA kit. Cells plated in 96-well plates were treated with samples and LPS as described in the sample treatment section. The appropriately diluted cell growth media were used to determine the amounts of $\mathrm{PGE}_{2}$ and each of the pro-inflammatory cytokines according to the manufacturer's instructions.

\section{Reverse Transcription-Polymerase Chain Reaction}

The effect of LGG and JW15 on the pro-inflammatory transcriptome was assessed in LPS-induced RAW 264.7 cells using an RT-PCR. Cells plated in 6-well culture plates were treated with samples and LPS as described in the sample treatment section. After rinsing twice with ice-cold PBS, total RNA was extracted with the RNeasy Mini Kit (Qiagen, Germany). Equal amounts $(1 \mu \mathrm{g})$ of total RNA were reverse-transcribed using a RevertAid First Strand cDNA Synthesis Kit. The mixtures containing synthesized cDNA, PCR mastermix, and $100 \mu \mathrm{M}$ of specific primers were amplified as follows: initial denaturation at $95^{\circ} \mathrm{C}$ for $5 \mathrm{~min}$ followed by $95^{\circ} \mathrm{C}$ for $30 \mathrm{sec}$, annealing at primer-specific temperature for $30 \mathrm{sec}$, extension at $72^{\circ} \mathrm{C}(20-25$ cycles $)$ and final extension at $72^{\circ} \mathrm{C}$ for $10 \mathrm{~min}$. The amplified PCR products were separated by electrophoresis on a $1.5 \%$ agarose gel containing DNA SafeStain (USA). Separated bands were visualized using a fluorescent table (Vilber Lourmat, France).

Relative quantification was conducted using real-time PCR (Thermo Scientific Pierce, USA). Using synthesized cDNA as a template, a PCR mixture containing SYBR Green PCR Master Mix and $400 \mu \mathrm{M}$ of specific primers was amplified as follows: $95^{\circ} \mathrm{C}$ for 2 min followed by 40 cycles at $95^{\circ} \mathrm{C}$ for $5 \mathrm{sec}, 60^{\circ} \mathrm{C}$ for $30 \mathrm{sec}$ with a final extension at $60^{\circ} \mathrm{C}$ for $30 \mathrm{sec}$. Single product amplification was verified with melting curve analysis at the end of the experiment. The result was analyzed after normalization with $\beta$ actin as a reference gene and calculated with the delta-delta $C_{t}$ $\left(\Delta \Delta C_{t}\right)$ method. Specific primer sequences used in this experiment are listed in Table 1.

\section{Western Blot Analysis}

The effect of LAB strains on the expression of pro-inflammatory proteins was determined in LPS-induced RAW 264.7 cells using a western blot analysis. Cells plated in 6-well culture plates were treated with samples and LPS as described in the sample treatment section. Rinsing twice with ice-cold PBS, cells were lysed with RIPA lysis buffer containing a protease and phosphatase inhibitor cocktail. Cell lysates were centrifuged $(13,000 \times g, 20 \mathrm{~min})$ and obtained supernatant was employed for analysis. Determining the total protein concentration of supernatant by using a $D C$ Protein Assay kit (Bio-Rad), equal

Table 1. Mouse-specific PCR primer sequences.

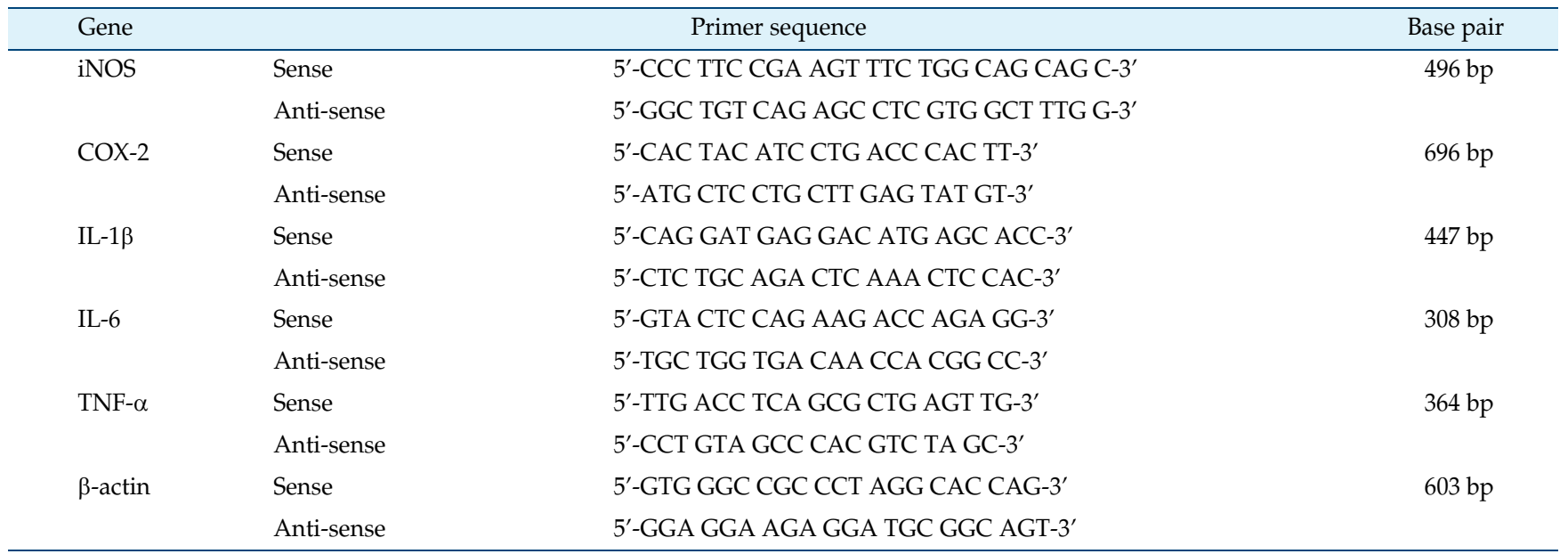


amounts $(30 \mu \mathrm{g})$ of total protein were separated by 8 or $12 \%$ of sodium dodecyl sulfate-polyacrylamide gel electrophoresis (SDSPAGE) and transferred onto polyvinylidene difluoride (PVDF) membranes. Transferred immunoblots were blocked with 5\% skim milk, and then probed with the primary antibodies $(1: 2,000)$ at $4^{\circ} \mathrm{C}$ for $16 \mathrm{~h}$. After brief washing with Tris-buffered saline containing Tween 20 (TBS-T), immunoblots were probed with secondary antibodies $(1: 5,000)$ at room temperature for $2 \mathrm{~h}$. After subsequent washing with TBS-T, blots were detected on an x-ray blue film by using an enhanced chemiluminescence reagent. Intensity of each band was measured with Image J software.

\section{Statistical Analysis}

The results are presented as the mean \pm standard deviation (SD) from independent experiments conducted in triplicates. Statistical analysis was performed using IBM SPSS for Windows version 18.0 (SPSS Inc., USA). The unpaired one-tailed Student's $t$-test was used to analyze statistical differences between two groups and one-way analysis of variance (ANOVA) was used to analyze statistical differences between multiple groups using Duncan's multiple-range test. $P$-values $<0.05$ were considered statistically significant.

\section{Results}

\section{Effect of Heat-Killed JW15 on Cell Viability}

To verify whether the anti-inflammatory properties were due to cytotoxicity, the effect of heat-killed JW15 on the cell viability was determined in RAW 264.7 cells. Neither heatkilled LGG nor JW15 had no significant effect on cell viability compared to either the LPS-positive groups or the LPS-negative groups at the tested concentrations (Fig. 1A). Therefore, regarding the anti-inflammatory effect of heatkilled JW15, the influence of cytotoxicity was not considered in subsequent experiments.

\section{Inhibition of $\mathrm{NO}$ and $\mathrm{PGE}_{2}$ Production}

To evaluate the anti-inflammatory potential, the effects of heat-killed JW15 on the production of NO and PGE were examined in LPS-induced RAW 264.7 cells. Compared to the LPS-negative group, treatment with LPS markedly increased the production of both $\mathrm{NO}$ and $\mathrm{PGE}_{2}$. However, treatment with heat-killed JW15 inhibited the production of $\mathrm{NO}$ and $\mathrm{PGE}_{2}$ in a dose-dependent manner (Figs. 1B and 1C). In addition, JW15 exhibited significant inhibitory effects on $\mathrm{NO}$ and $\mathrm{PGE}_{2}$ production compared to LGG at the tested levels.

\section{Suppression of iNOS and COX-2 Expression}

To determine whether the inhibitory effect of JW15 on $\mathrm{NO}$ and $\mathrm{PGE}_{2}$ production was associated with changes in
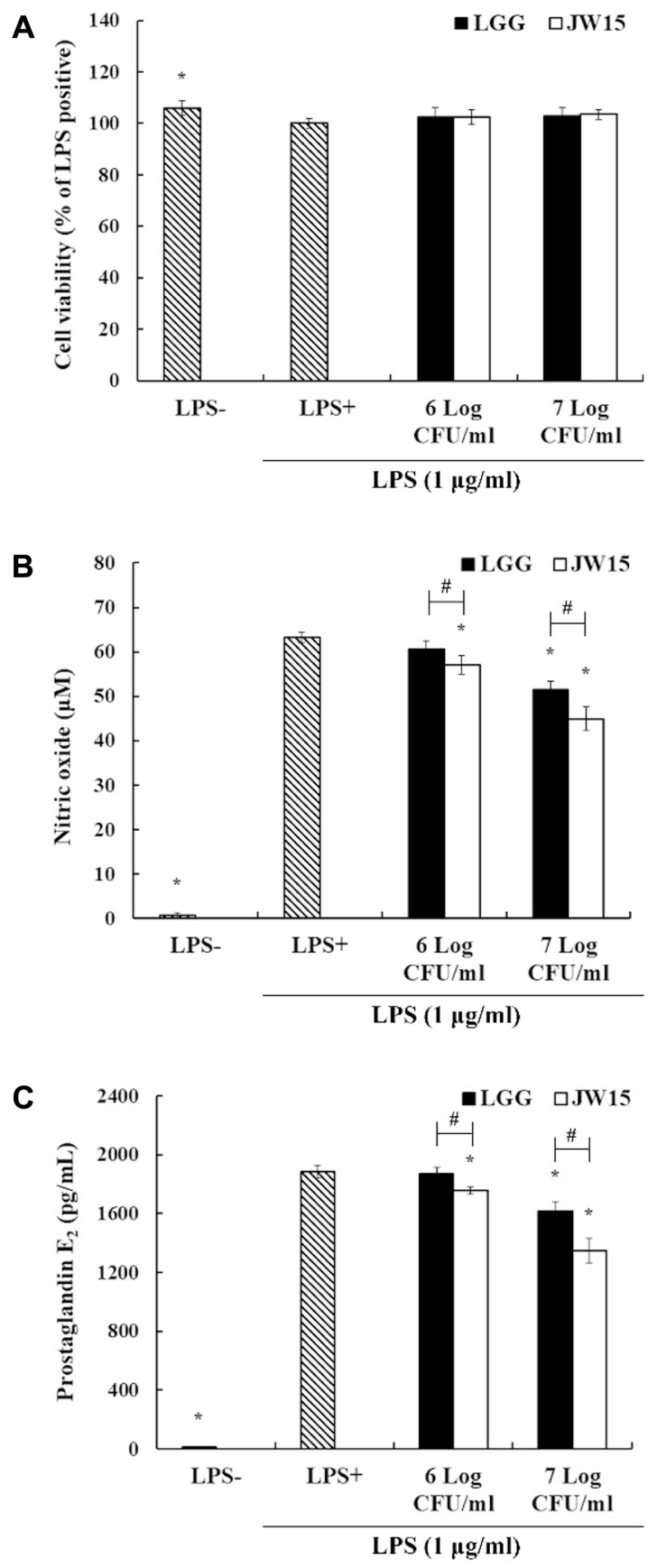

Fig. 1. Effect of heat-killed LAB strains on (A) cell viability and production of (B) $\mathrm{NO}$ and $(\mathbf{C}) \mathrm{PGE}_{2}$ in LPS-induced RAW 264.7 cells.

Cells were treated with heat-killed LGG or JW15 and stimulated with LPS for $24 \mathrm{~h}$. The results were expressed as mean \pm S.D. of three independent experiments. ${ }^{*} p<0.05$, compared to LPS positive group; $\# p<0.05$, comparison between LGG and JW15.

the expression levels of their respective synthesis enzymes, protein and transcriptional expression levels of iNOS and 
A
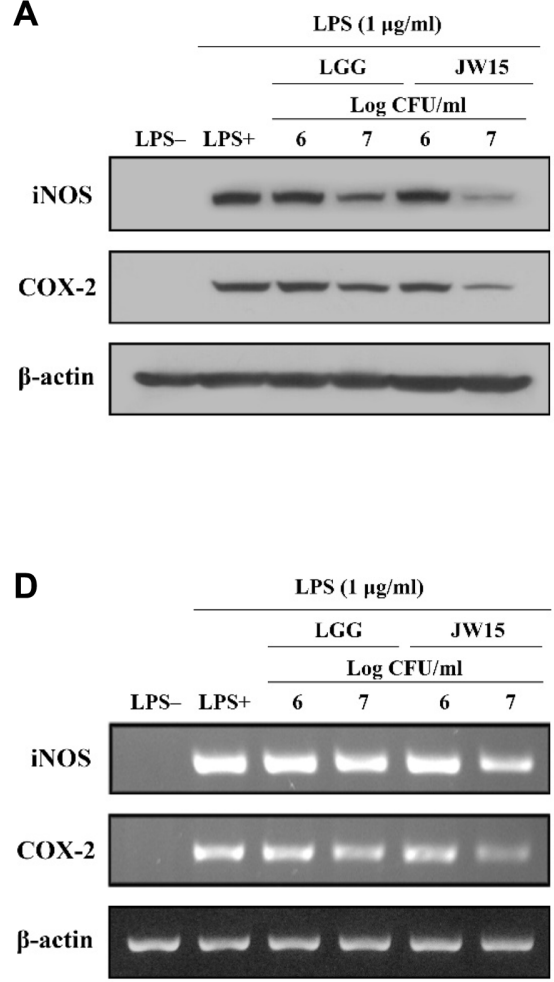

B

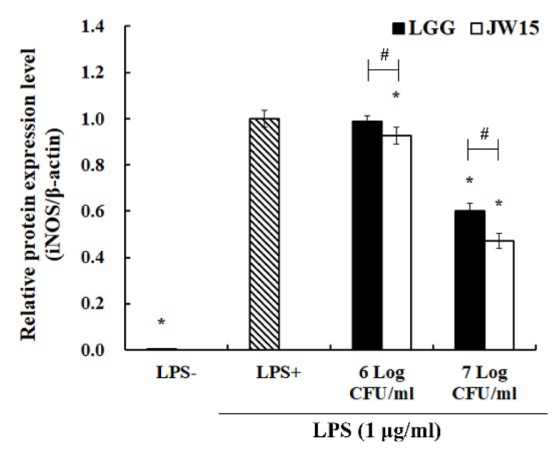

E

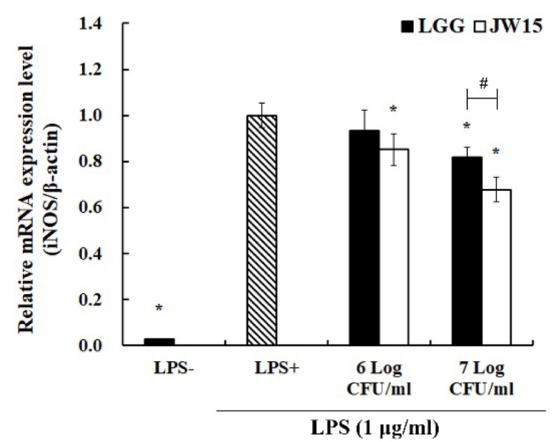

C

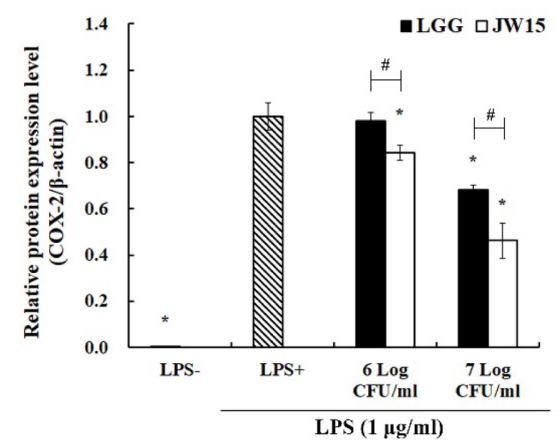

$\mathbf{F}$

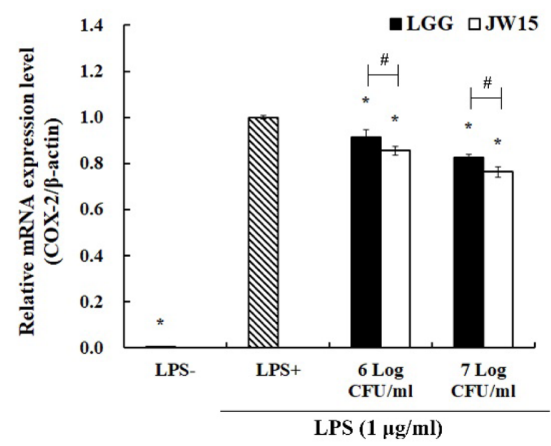

Fig. 2. Suppressive effect of heat-killed LAB strains on the expression of iNOS and COX-2 in LPS-induced RAW 264.7 cells. Cells were treated with heat-killed LGG or JW15 and stimulated with LPS for $24 \mathrm{~h}$. Expressed protein levels of iNOS and COX-2 were determined with (A) immunoblots and (B and C) relative quantification of band intensity. Expressed mRNA levels of iNOS and COX-2 were determined with (D) agarose gel blotting and $(\mathbf{E}$ and $\mathbf{F})$ relative quantification by normalization with $\beta$-actin. The results were expressed as mean \pm S.D. of three independent experiments. ${ }^{*} p<0.05$, compared to LPS positive group; $\# p<0.05$, comparison between LGG and JW15.

COX-2 were investigated. As expected, treatment with LPS markedly increased the protein expression levels of iNOS and COX-2. However, treatment with heat-killed JW15 suppressed the expression levels of iNOS and COX-2 (Figs. 2A-2C). Similarly, the mRNA levels of iNOS and COX-2 were up-regulated by LPS stimulation and were down-regulated following treatment with heat-killed JW15 (Figs. 2D-2F). This result indicated that treatment of JW15 inhibits production of $\mathrm{NO}$ and $\mathrm{PGE}_{2}$ through suppressing the expression of iNOS and COX-2. Further, JW15 exhibited significant inhibitory effects against both iNOS and COX-2 expression compared to LGG at the tested levels.

\section{Suppressive Effect on Pro-Inflammatory Cytokine Expression}

JW15-mediated effect on expression levels of proinflammatory cytokines was examined in LPS-induced
RAW 264.7 cells. Compared to the LPS-negative group, treatment of LPS markedly increased the production of IL$1 \beta$, IL-6, and TNF- $\alpha$. However, the concentration of those pro-inflammatory cytokines was decreased following the treatment of heat-killed JW15 in a dose-dependent manner (Figs. 3A-3C). Consistent with the results of the ELISA, transcriptional expression of IL-1 $\beta$, IL- 6 , and TNF- $\alpha$ was significantly induced by stimulation of LPS, contrarily, mRNA expression levels of those pro-inflammatory cytokines were attenuated by treatment of heat-killed JW15 (Figs. 3D-3G). Moreover, JW15 exhibited significant inhibitory effect on expression of pro-inflammatory cytokines compared to LGG at a concentration of 7 Log $\mathrm{CFU} / \mathrm{ml}$. Consequently, the down-regulated proinflammatory signatures following treatment with heatkilled JW15 in LPS-induced RAW 264.7 cells led to the hypothesis that JW15-mediated anti-inflammatory properties might be associated with regulation of NF- $\mathrm{BB}$ pathway. 

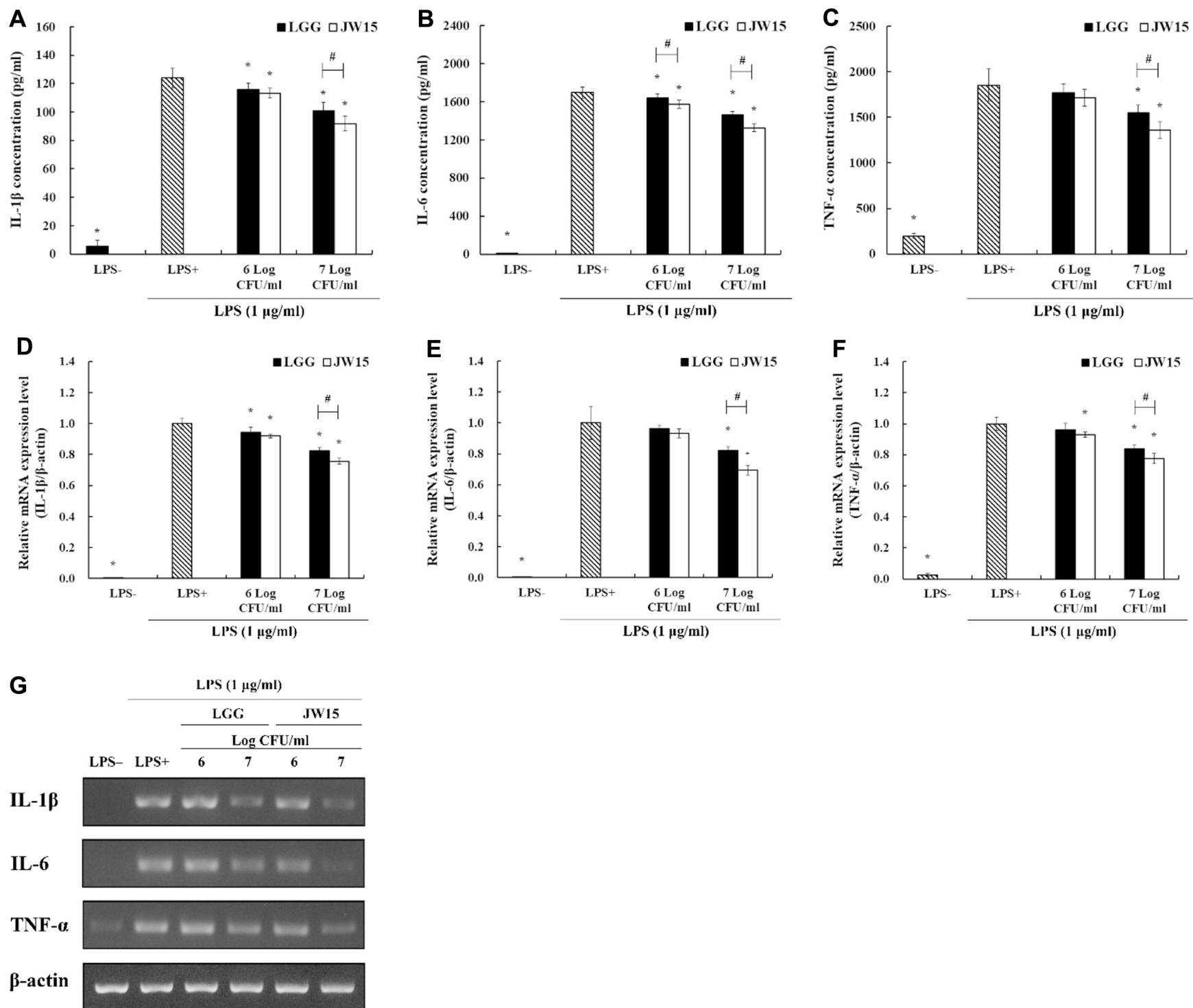

Fig. 3. Down-regulatory effect of heat-killed LAB strains on the expression of pro-inflammatory cytokines in LPS-induced RAW 264.7 cells.

Cells were treated with heat-killed LGG or JW15 and stimulated with LPS for $24 \mathrm{~h}$. The amounts of IL-1 $\beta$, IL-6, and TNF- $\alpha$ were assessed with (AC) an ELISA kit. Expressed mRNA levels of those cytokines were determined with (D-F) relative quantification by normalization with $\beta$-actin and (G) agarose gel blotting. The results were expressed as mean \pm S.D. of three independent experiments. ${ }^{*} p<0.05$, compared to LPS positive group; $\# p<0.05$, comparison between LGG and JW15.

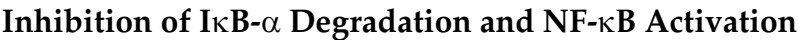

To verify whether the down-regulated expression of proinflammatory factors was associated with alteration of transcription factors, the effect of heat-killed JW15 on the activation of NF- $\mathrm{BB}$ was investigated in LPS-induced RAW 264.7 cells. Treatment with LPS significantly induced the phosphorylation of I $\mathrm{I} \mathrm{B}-\alpha$ indicating the degradation of

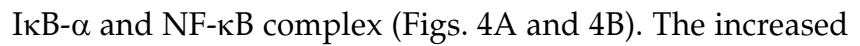
phosphorylation of I $\mathrm{B}-\alpha$ was correlated with up-regulated phosphorylation of p65, a subunit of the NF- $\kappa B$ heterodimer (Figs. 4A and 4C). However, pre-treatment with heat-killed JW15 decreased the degradation of IкB- $\alpha$ and NF- $\kappa$ B complex by inhibiting the phosphorylation of IкB- $\alpha$ and p65 (Figs. 4A-4C). In addition, JW15 exhibited a 


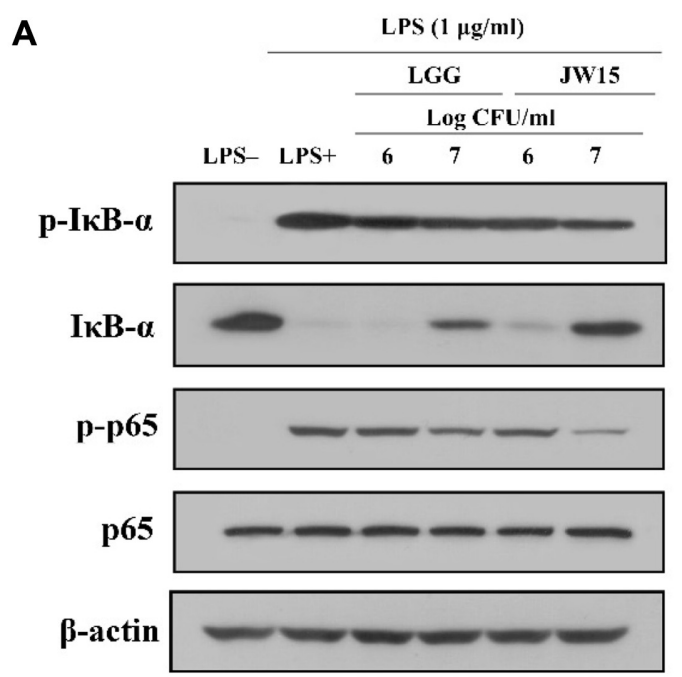

B

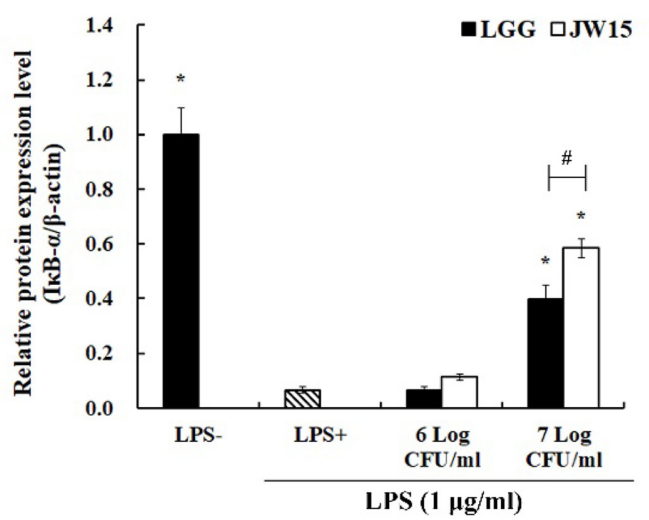

C

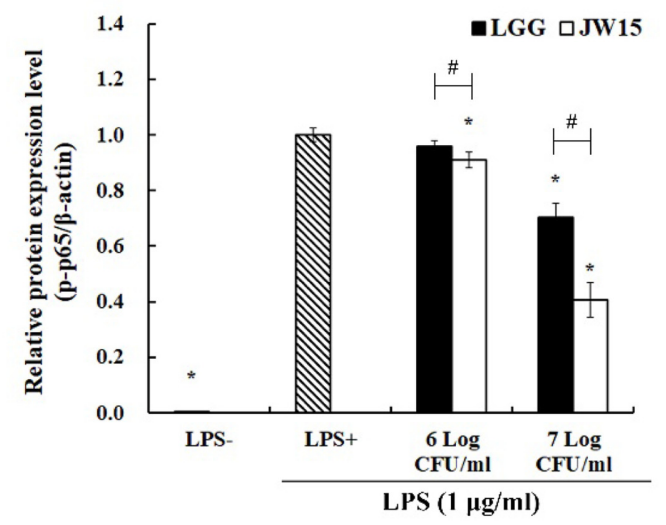

Fig. 4. Inhibitory effect of heat-killed LAB strains on the NF-кB activation in LPS-induced RAW 264.7 cells.

Cells were treated with heat-killed LGG or JW15 and stimulated with LPS for $30 \mathrm{~min}$. Alteration in protein expression of IкB- $\alpha$ and NF-кB was evaluated with (A) Immunoblots and (B and C) relative quantification of band intensity. The results were expressed as mean \pm S.D. of three independent experiments. ${ }^{*} p<0.05$, compared to LPS positive group; $\# p<0.05$, comparison between LGG and JW15.

significant inhibitory effect on NF-kB activation compared to LGG at the tested levels.

\section{Inhibition of MAPKs Activation}

To gain more insight into the anti-inflammatory properties of JW15, the effect of heat-killed JW15 on MAPKs activation was examined in RAW 264.7 cells upon LPS stimulation. As expected, phosphorylation of MAPKs including ERK 1/2, JNK and p38 was markedly increased following LPS stimulation. However, treatment of heat-killed JW15 suppressed phosphorylation of MAPKs in a dose-dependent manner (Figs. 5A-5D). These results suggested that JW15mediated anti-inflammatory properties are associated with inhibition of MAPKs activation. Furthermore, JW15 displayed a significant suppressing effect on MAPKs activation compared to LGG, except for JNK.

\section{Discussion}

The beneficial effects of probiotics are primarily derived from the GIT, because it contains the largest proportion of human microbiota [8]. The cells of the GIT, including intestinal epithelial cells, lymphocytes, dendritic cells, and macrophages, possess various pattern recognition receptors (PRRs) that recognize the conserved microbe-associated molecular patterns (MAMPs). Various MAMPs in bacterial surface molecules, including peptidoglycan, LPS, and lipoteichoic acid (LTA), interact with PRRs and directly or indirectly mediate the cellular physiological functions, such as immune responses, differentiation, and proliferation [17, 18]. Composition of bacterial surface molecules such as amino acid residues, disaccharide ratio, and difference in cross-link type, is different between microbes, thereby, 

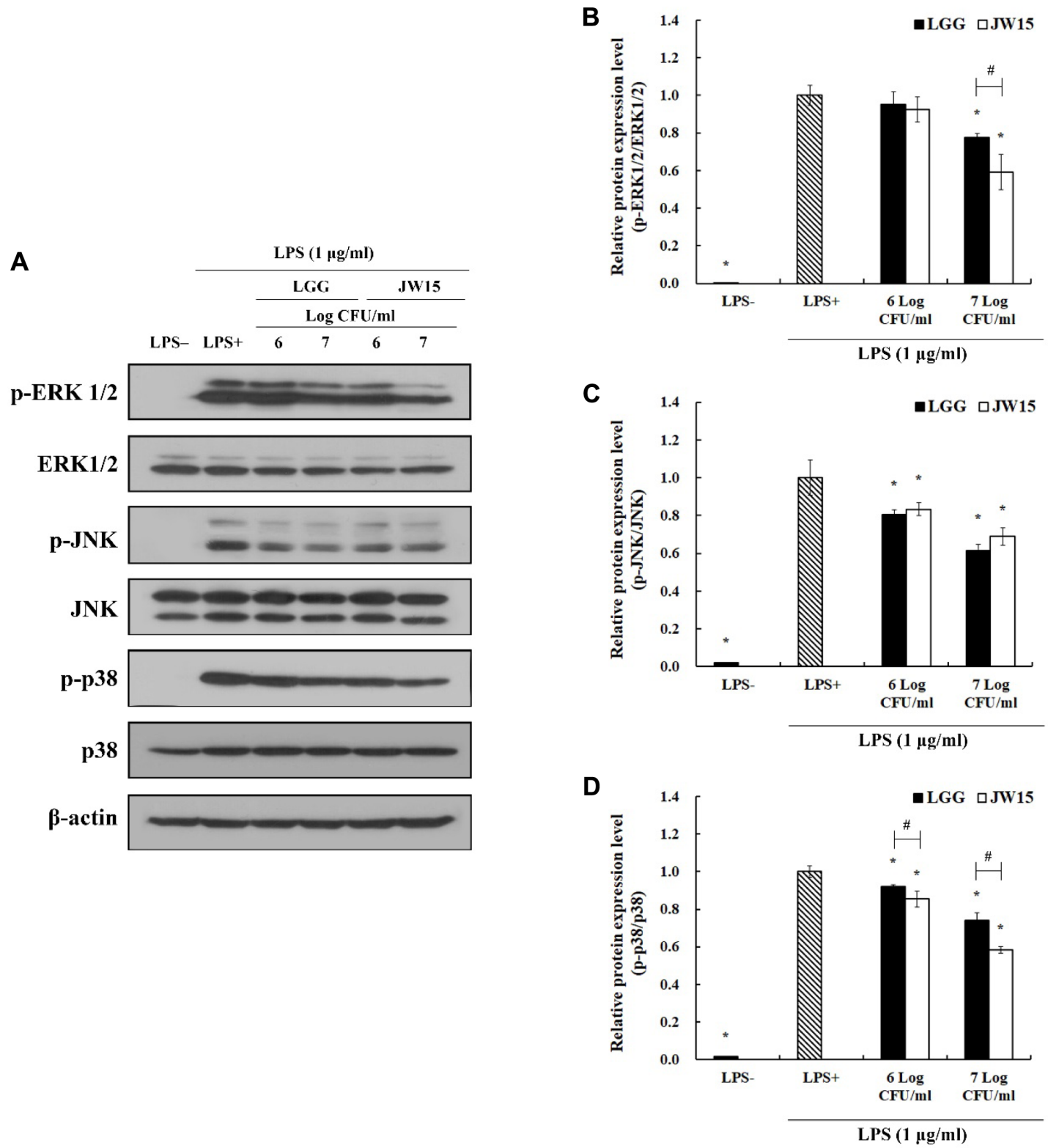

Fig. 5. Inhibitory effect of heat-killed LAB strains on the MAPKs activation in LPS-induced RAW 264.7 cells.

Cells were treated with heat-killed LGG or JW15 and stimulated with LPS for $30 \mathrm{~min}$. Protein expression levels of MAPKs were determined with (A) Immunoblots and (B-D) relative quantification of band intensity. The results were expressed as mean \pm S.D. of three independent experiments. ${ }^{*} p<0.05$, compared to LPS positive group; $\# p<0.05$, comparison between LGG and JW15.

microbe-mediated immune responses are depend on specificity of bacterial strains $[19,20]$.

The inflammatory response is a multi-cascade defensive process against external invasion, including elimination of stimuli and initiation of recovery processes, and is therefore crucial for maintaining health [21]. Macrophages play an essential role in the inflammatory responses by perceiving the foreign threats with specific receptors, including the Toll-like receptor (TLR)-4 [22]. Upon recognition of LPS by TLR-4, macrophages initiate extensive inflammatory responses, including activation of MAPKs and NF- $\mathrm{\kappa B}$ resulting in up-regulated production of proinflammatory mediators such as cytokines, $\mathrm{NO}$, and $\mathrm{PGE}_{2}$. However, excessive or prolonged expression of proinflammatory mediators causes immune system imbalance and may result in acute or chronic inflammatory disorders 
such as arthritis, asthma, IBD, and multiple sclerosis [23]. Moreover, suppression of excessive pro-inflammatory mediators derived from aberrantly activated macrophages may lead to attenuation of inflammation-induced disorders [24]. The current study was therefore performed to evaluate the anti-inflammatory potential of JW15 and to elucidate the associated cellular signaling pathways.

$\mathrm{NO}$ and prostaglandins are modulated by distinct NOS and COX isoforms, respectively, and are important biomarkers in the inflammatory responses that are inducing the pain, swelling, fever, and tenderness [25]. Among the isoforms of those enzymes, iNOS and COX-2 are undetectable in the basal state. However, specific stimuli, including LPS, induce the expression of those enzymes through NF- $\mathrm{kB}$ activation and result in excessive production of $\mathrm{NO}$ and $\mathrm{PGE}_{2}$. Inordinate generation of $\mathrm{NO}$ and $\mathrm{PGE}_{2}$ causes up-regulated expression levels of other pro-inflammatory mediators, including the cytokines, and could lead to malfunctions ranging from severe cell injury to inflammatory disorders [26]. Therefore, regulating the iNOS and COX-2 is one of the most critical strategies for developing therapeutic materials for inflammatory diseases. In the current study, treatment with heat-killed JW15 decreased the production of $\mathrm{NO}$ and $\mathrm{PGE}_{2}$ in RAW 264.7 cells against challenge of LPS via suppressing the iNOS and COX-2 at both the mRNA and protein levels. Similarly, treatment of heat-killed Lactobacillus brevis K65 decreased the production of $\mathrm{NO}$ and $\mathrm{PGE}_{2}$ in RAW 264.7 cells upon LPS stimulation, which is attributable to downregulated expression levels of iNOS and COX-2 [27].

Inflammatory responses to LPS were accompanied by markedly up-regulated production of pro-inflammatory cytokines. Synthesis of pro-inflammatory cytokines, including IL- $1 \beta$, IL- 6 , and TNF- $\alpha$, is primarily mediated by COX-2 resulting from NF-KB activation and excessive production of those cytokines has been known to be associated with occurrence of both acute and chronic inflammation $[28,29]$. Thus, alleviation of pro-inflammatory cytokines is important for improving inflammatory disorders. For instance, over-expressed IL-6 has been known to play a pivotal role in the development of rheumatoid arthritis and Th1 cell-mediated chronic colitis, however, the IL-6 gene-knockout mice are resistant to dextran sodium sulphate-induced colitis. Consistent with these findings, heat-killed L. casei Shirota-mediated attenuation of IBD was involved in the inhibition of the IL6/STAT-3 signaling pathway [30]. Additionally, W. cibaria inhibited production of IL-6 and IL-8 in Fusobacterium nucleatum-induced KB cells [13]. Therefore, the current results indicated that JW15-mediated modulatory effect on the expression of the pro-inflammatory cytokines potentially contributes to improvement of the inflammatory disorders as a clinical application.

NF- $\kappa \mathrm{B}$ is a primary transcriptional factor modulating the expression of pro-inflammatory genes. Recognition of LPS

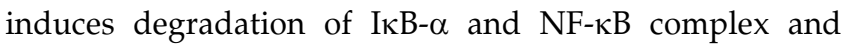
results in translocation of NF- $\mathrm{kB}$ to the nucleus. Translocated NF- $\mathrm{KB}$ initiates the transcription of iNOS, COX-2, and pro-inflammatory cytokines [22, 24]. It has been found that excessively activated NF-kB is strongly associated with inflammatory diseases. Accordingly, NF$\kappa \mathrm{B}$-mediated over-expression of cytokines and adhesion molecules has been known to be involved in pathogenicity of inflammatory airway diseases, a result that correlates with the inordinate activation of NF- $\mathrm{kB}$ found in bronchitis biopsies of asthma patients [26, 31]. The present study demonstrated that down-regulated pro-inflammatory features following treatment with heat-killed JW15 in LPSinduced RAW 264.7 cells were associated with suppressing the activation of NF- $\kappa$ B. These results are supported by much experimental evidence demonstrating the probioticsmediated improvement of inflammatory disorders. Previous study demonstrated that LGG attenuates the interferon- $\gamma$ and TNF- $\alpha$-induced intestinal barrier malfunction by attenuating the pro-inflammatory responses resulting from the suppression of MAPKs and NF-kB activation [32]. Similarly, Lactobacillus suntoryeus HY7801 displayed downregulated the expression of pro-inflammatory cytokines through inhibiting the TLR-4-linked activation of NF- $\mathrm{kB}$ in 2,4,6-trinitrobenzenesulfonic acid-induced colitic mice [33]. Collectively, strategies suppressing the aberrant activation of NF- $\mathrm{KB}$ are pivotal in the development of therapeutic approaches regarding the improvement of inflammatory disorders.

The MAPKs are part of an important intracellular signaling pathway for transcriptional regulation of proinflammatory responses through NF-KB activation [23]. Although the precise signaling pathways of MAPKs remain unclear, the present study showed that treatment with heat-killed JW15 suppresses the activation of MAPKs, ERK $1 / 2$, JNK, and p38, in a dose-dependent manner. Similarly, several studies have demonstrated that LPS-induced activation of MAPKs results in initiation of inflammatory responses including pro-inflammatory factors. Amongst three subfamilies of MAPKs, only p38 has been linked with the expression of iNOS against stimulation of LPS [3, 34]. As a probiotic application, L. casei OLL2768 down-regulated Escherichia coli-mediated inflammatory signatures through 
suppressing the MAPKs activation in a bovine epithelial cell line [35]. Additionally, Lactobacillus pentosus b240 alleviated Streptococcus pneumoniae-induced pneumonia by modulating the expression of MAPKs, and of specific PRRs including TLR-2 and TLR-4 [36].

In conclusion, the present study demonstrated the antiinflammatory potential of JW15 derived from kimchi as a functional probiotic candidate. Heat-killed JW15 displayed anti-inflammatory potential by alleviating the proinflammatory features in LPS-induced RAW 264.7 cells through suppressing NF- $\mathrm{KB}$ activation. The cellular signaling pathways of its anti-inflammatory effect were involved in inhibition of MAPKs activation. Although further in vivo investigation is still required to confirm the anti-inflammatory effect of JW15, these results may provide the molecular evidence for the immunomodulatory effect of probiotics. Taken together, JW15 might be employed for development of functional probiotic products in the food and pharmaceutical industries.

\section{Acknowledgments}

This research was carried out with financial support from the Cooperative Research Program for Agriculture Science and Technology Development, Rural Development Administration, Republic of Korea (Project No. PJ01254102).

\section{Conflict of Interest}

The authors have no financial conflicts of interest to declare.

\section{References}

1. Lee SH, Kwak CH, Lee SK, Ha SH, Park J, Chung TW, et al. 2016. Anti-inflammatory effect of ascochlorin in LPS-stimulated RAW 264.7 macrophage cells is accompanied with the down-regulation of iNOS, COX-2 and proinflammatory cytokines through NF- $\mathrm{B}$, ERK1/2, and p38 signaling pathway. J. Cell. Biochem. 117: 978-987.

2. Ran S, Montgomery KE. 2012. Macrophage-mediated lymphangiogenesis: the emerging role of macrophages as lymphatic endothelial progenitors. Cancers (Basel) 4: 618-657.

3. Yu GJ, Choi IW, Kim GY, Kim BW, Park C, Hong SH, et al. 2015. Anti-inflammatory potential of saponins derived from cultured wild ginseng roots in lipopolysaccharide-stimulated RAW 264.7 macrophages. Int. J. Mol. Med. 35: 1690-1698.

4. Glushkova OV, Parfenyuk SB, Khrenov MO, Novoselova TV, Lunin SM, Fesenko EE, et al. 2013. Inhibitors of TLR-4, $\mathrm{NF}-\mathrm{KB}$, and SAPK/JNK signaling reduce the toxic effect of lipopolysaccharide on RAW 264.7 cells. J. Immunotoxicol. 10: 133-140.

5. Ou CC, Lin SL, Tsai JJ, Lin MY. 2011. Heat-killed lactic acid bacteria enhance immunomodulatory potential by skewing the immune response toward Th1 polarization. J. Food Sci. 76: M260-M267.

6. Khan I, Kang SC. 2016. Probiotic potential of nutritionally improved Lactobacillus plantarum DGK-17 isolated from kimchi - A traditional Korean fermented food. Food Control 60: $88-94$.

7. Feleszko W, Jaworska J, Rha RD, Steinhausen S, Avagyan A, Jaudszus A, et al. 2007. Probiotic-induced suppression of allergic sensitization and airway inflammation is associated with an increase of $\mathrm{T}$ regulatory-dependent mechanisms in a murine model of asthma. Clin. Exp. Allergy 37: 498-505.

8. Lebeer S, Claes IJ, Vanderleyden J. 2012. Anti-inflammatory potential of probiotics: lipoteichoic acid makes a difference. Trends Microbiol. 20: 5-10.

9. Sashihara T, Sucki N, Ikegami S. 2006. An analysis of the effectiveness of heat-killed lactic acid bacteria in alleviating allergic diseases. J. Dairy Sci. 89: 127-133.

10. Li N, Russell WM, Douglas-Escobar M, Hauser N, Lopez M, Neu J. 2009. Live and heat-killed Lactobacillus rhamnosus GG: effect on proinflammatory and anti-inflammatory cytokines/ chemokines in gastrostomy-fed infant rats. Pediatr. Res. 66: 203-207.

11. Won TJ, Kim B, Song DS, Lim YT, Oh ES, Lee DI, et al. 2011. Modulation of Th1/Th2 balance by Lactobacillus strains isolated from Kimchi via stimulation of macrophage cell line J774A.1 in vitro. J. Food Sci. 76: H55-H61.

12. Son SH, Jeon HL, Jeon EB, Lee NK, Park YS, Paik, HD. 2017. Potential probiotic Lactobacillus plantarum Ln4 from kimchi: Evaluation of $\beta$-galactosidase and antioxidant activities. LWT-Food Sci. Technol. 85: 181-186.

13. Kang MS, Lim HS, Kim SM, Lee HC, Oh JS. 2011. Effect of Weissella cibaria on Fusobacterium nucleatum-induced interleukin-6 and interleukin-8 production in KB cells. J. Bacteriol. Virol. 41: 9-18.

14. Lee WK, Ahn SB, Park HE, Lee SM, Kim SY, Shon MY. 2013. Characteristics and immuno-modulatory effects of Weissella cibaria JW15 isolated from Kimchi, Korea traditional fermented food, for probiotic use. J. Biomed. Res. 14: 206-211.

15. Yu HS, Lee NK, Choi AJ, Choe JS, Bae CH, Paik HD. 2019. Antagonistic and antioxidant effect of probiotic Weissella cibaria JW15. Food Sci. Biotechnol. 28: 851-855.

16. Kim HS, Yu HS, Lee JH, Lee GW, Choi SJ, Chang PS, et al. 2018. Application of stabilizer improves stability of nanosuspended branched-chain amino acids and antiinflammatory effect in LPS-induced RAW 264.7 cells. Food Sci. Biotechnol. 27: 451-459.

17. Medzhitov R. 2007. Recognition of microorganisms and activation of the immune response. Nature 449: 819-826. 
18. Lebeer S, Vanerleyden J, Keersmaecker SCJ. 2010. Host interactions of probiotic bacterial surface molecules: comparison with commensals and pathogens. Nat. Rev. Microbiol. 8: 171-184.

19. Wu Z, Pan D, Guo Y, Sun Y, Zeng X. 2015. Peptidoglycan diversity and anti-inflammatory capacity in Lactobacillus strains. Carbohydr. Polym. 128: 130-137.

20. Jeong JH, Jang S, Jung BJ, Jang KS, Kim BG, Chung DK, et al. 2014. Differential immune-stimulatory effects of LTAs from different lactic acid bacteria via MAPK signaling pathway in RAW 264.7 cells. Immunobiology 220: 460-466.

21. Mariathasan S, Monack DM. 2007. Inflammasome adaptors and sensors: intracellular regulators of infection and inflammation. Nat. Rev. Immunol. 7: 31-40.

22. Kim KN, Heo SJ, Yoon WJ, Kang SM, Ahn G, Yi TH, et al. 2010. Fucoxanthin inhibits the inflammatory response by suppressing the activation of NF- $\mathrm{KB}$ and MAPKs in lipopolysaccharide-induced RAW 264.7 macrophages. Eur. J. Pharmacol. 649: 369-375.

23. Fernando IS, Sanjeewa KA, Samarakoon KW, Lee WW, Kim HS, Ranasinghe P, et al. 2018. Antioxidant and antiinflammatory functionality of ten Sri Lankan seaweed extracts obtained by carbohydrase assisted extraction. Food Sci. Biotechnol. 27: 1761-1769.

24. Lawrence T, Willoughby DA, Gilroy DW. 2002. Antiinflammatory lipid mediators and insights into the resolution of inflammation. Nat. Rev. Immunol. 2: 787-795.

25. Hu SS, Bradshaw HB, Chen JS, Tan B, Walker JM. 2008. Prostaglandin $\mathrm{E}_{2}$ glycerol ester, an endogenous COX-2 metabolite of 2-arachidonoylglycerol, induces hyperalgesia and modulates NFKB activity. Br. J. Pharmacol. 153: 15381549.

26. Tak PP, Firestein GS. 2001. NF-кB: a key role in inflammatory diseases. J. Clin. Invest. 107: 7-11.

27. Liu YW, Ong WK, Su YW, Hsu CC, Cheng TH, Tsai YC. 2016. Anti-inflammatory effects of Lactobacillus brevis $\mathrm{K} 65$ on RAW 264.7 cells and in mice with dextran sulphate sodiuminduced ulcerative colitis. Benef. Microbes 7: 387-396.
28. Aupperle KR, Bennett BL, Boyle DL, Tak PP, Manning AM, Firestein GS. 1999. NF-кB regulation by IкB kinase in primary fibroblast-like synoviocytes. J. Immunol. 163: 427-433.

29. Atreya R, Mudter J, Finotto S, Müllberg J, Jostock T, Wirtz $S$, et al. 2000. Blockade of interleukin 6 trans signaling suppresses T-cell resistance against apoptosis in chronic intestinal inflammation: Evidence in Crohn diseases and experimental colitis in vivo. Nat. Med. 6: 583-588.

30. Matsumoto S, Hara T, Hori T, Mitsuyama K, Nagaoka M, Tomiyasu N, et al. 2005. Probiotic Lactobacillus-induced improvement in murine chronic inflammatory bowel disease is associated with the down-regulation of pro-inflammatory cytokines in lamina propria mononuclear cells. Clin. Exp. Immunol. 140: 417-426.

31. Hart LA, Krishnan VL, Adcock IM, Barnes PJ, Chung KF. 1999. Activation and localization of transcription factor, nuclear factor-кВ, in asthma. Am. J. Respir. Crit. Care Med. 158: $1585-1592$

32. Donato KA, Gareau MG, Wang YJJ, Sherman, PM. 2010. Lactobacillus rhamnosus GG attenuates interferon- $\gamma$ and tumor necrosis factor- $\alpha$-induced barrier dysfunction and pro-inflammatory signalling. Microbiology 156: 3288-3297.

33. Lee JH, Lee B, Lee HS, Bae EA, Lee H, Ahn YT, et al. 2008. Lactobacillus suntoryeus inhibits pro-inflammatory cytokine expression and TLR-4-linked NF- $\mathrm{KB}$ activation in experimental colitis. Int. J. Colorectal Dis. 24: 231-237.

34. Chen CC, Wang JK, 1999. P38 but not p44/42 mitogenactivated protein kinase is required for nitric oxide synthase induction mediated by lipopolysaccharide in RAW 264.7 macrophages. Mol. Pharmacol. 55: 481-488.

35. Takanashi N, Tomosada Y, Villena J, Murata K, Takahashi T, Chiba E, et al. 2013. Advanced application of bovine intestinal epithelial cell line for evaluation regulatory effect of lactobacilli against heat-killed enterotoxigenic Escherichia coli-mediated inflammation. BMC Microbiol. 13: 54.

36. Tanaka A, Seki M, Yamahira S, Noguchi H, Kosai K, Toba M, et al. 2011. Lactobacillus pentosus strain b240 suppresses pneumonia induced by Streptococcus pneumoniae in mice. Lett. Appl. Microbiol. 53: 35-43. 